\title{
EL ABASTECIMIENTO DE SEVILLA Y SU ZONA DE INFLUENCIA: INERCIAS DE LA POLÍTICA DE OFERTA Y DEBILIDADES DE LA GESTIÓN DE LA DEMANDA
}

\author{
Leandro del Moral Ituarte
}

\section{RESUMEN}

Justamente al finalizar la Exposición Universal de 1992, el sistema de abastecimiento de Sevilla entra en crisis. El análisis de esta situación obliga a tomar en consideración, al menos, seis factores, cuyo tratamiento puede ser de utilidad para el enfoque más general del problema de los abastecimientos en España: los métodos de evaluación de los recursos disponibles, las características de la gestión del sistema (modelos de consumo, estructura tarifaria y gestión de la red de distribución), las posibilidades de reasignación de recursos entre usuarios, la potencialidad del uso integrado de aguas superficiales y subterráneas, los costes ambientales de las soluciones propuestas y el tratamiento de la información y educación de los usuarios. Al final, se avanzan las condiciones que, en opinión de autor, un programa de gestión de la demanda, basado en la planificación al mínimo coste, habría de cumplir.

\section{SUMMARY}

Just at the end of the Expo 92, the water supply system of Seville breaks down. In order to analyse this issue, we must become aware of, at least, six aspects whose examination could help to understand the problems of water supply systems that affect the whole country: the assesment of the available resources, the management features (water-use patterns, rate system and water conservation practices), the possibilities of conversion of irrigation water supplies to meet domestic demands, the advantage of integrated management of ground and surface water, the environmental costs of the alternative solutions and the treatment of the consumers information and education. Last, the author suggests the minimal requirements that a demand management program, based in a integrated resource least cost planning, should fulfil.

\section{Introducción}

La situación del abastecimiento de agua a Sevilla y su zona de influencia ha atravesado a lo largo del año 1993 el momento más crítico de su historia reciente. Si bien el problema 
de desabastecimiento en términos absolutos ha sido resuelto por medio del recurso a las aguas del Guadalquivir, la población ha rechazado como inapropiadas para el consumo unas aguas que incumplen algunos de los parámetros de calidad (conductividad y cloruros) establecidos por la normativa vigente.

Los problemas del abastecimiento de agua a Sevilla no son nuevos, sino que forman parte consustancial de su historia. A finales del siglo pasado, la ciudad tuvo que sustituir el abastecimiento tradicional, que se basaba en las fuentes de Tomares, del Arzobispo y, sobre todo, en los manantiales de Santa Lucía, situados en Alcalá de Guadaira y canalizados hasta la ciudad a través de los Caños de Carmona. Pocas décadas después, también la conducción de «los Ingleses», basada de nuevo en manantiales de Alcalá de Guadaira - Zacatín, La Judía, Fuensanta, Retama, Las Aceñas y Otivar- ayudados por los de Clavinque, en el término de Mairena del Alcor, resultó insufiente y hubo que recurrir a las aguas filtradas del Guadalquivir, que se distribuían por una red separada y no se dedicaban al consumo de boca (MORAL ITUARTE, 1991).

Sin embargo, pese a estos precedentes, la crisis (colapso durante 1993) del moderno sistema basado en el aprovechamiento del río Rivera de Huelva es de una magnitud extraordinaria y, como vamos a ver, imprevista e insospechada.

La situación del abastecimiento de Sevilla y su área de influencia plantea algunos temas que trascienden los límites del caso concreto y ofrece elementos de interés para la consideración de la problemática general de la gestión de los abastecimientos urbanos en España:

1. En primer lugar, un tema de carácter estrictamente hidrológico: el cálculo de los recursos disponibles. En este caso, al tratarse de un sistema basado exclusivamente en el aprovechamiento de aguas superficiales, este problema tiene que ver con la evaluación de la capacidad de regulación de los embalses, teniendo en cuenta los criterios de garantía - déficits admitidos - adecuados al tipo de demanda de que se trata, es decir, a los usos urbanos.

2. En segundo lugar, destacan los aspectos relacionados con la gestión de la demanda, que se refieren a problemas tan esenciales como la evolución de los modelos de consumo, la estructura tarifaria y la gestión de la red de distribución.

3. En tercer lugar, la situación actual del abastecimiento de Sevilla ofrece un ejemplo paradigmático de uno de los grandes temas que la planificación hidrológica española tiene planteados en la actualidad. Nos referimos al problema de la reasignación de recursos entre antiguos y nuevos usuarios, y particularmente entre usos agrícolas que cuentan con sus correspondientes concesiones desde hace décadas y nuevas demandas urbanas insuficientemente abastecidas en cantidad o calidad.

4. En cuarto lugar, la opción por un sistema de abastecimiento basado exclusivamente, como queda dicho, en las aguas superficiales remite al tema de las posibilidades de mejorar la gestión del agua con la integración de las dos fases del ciclo hidráulico, recuperando, por tanto, las potencialidades de los recursos subterráneos. Esta cuestión tiene la máxima vigencia en los debates actuales sobre planificación y gestión del agua.

5. En quinto lugar, las diferentes soluciones ofrecidas a la crisis plantean el problema de los costes ambientales que una estrategia basada en la ampliación de la capacidad de embalse pudiera acarrear.

6. Por último, y en otro orden de cosas, la situación del abastecimiento a Sevilla ofrece una buena ocasión para discutir el tratamiento de las cuestiones referidas a información, educación del consumo y coordinación de las administraciones. 


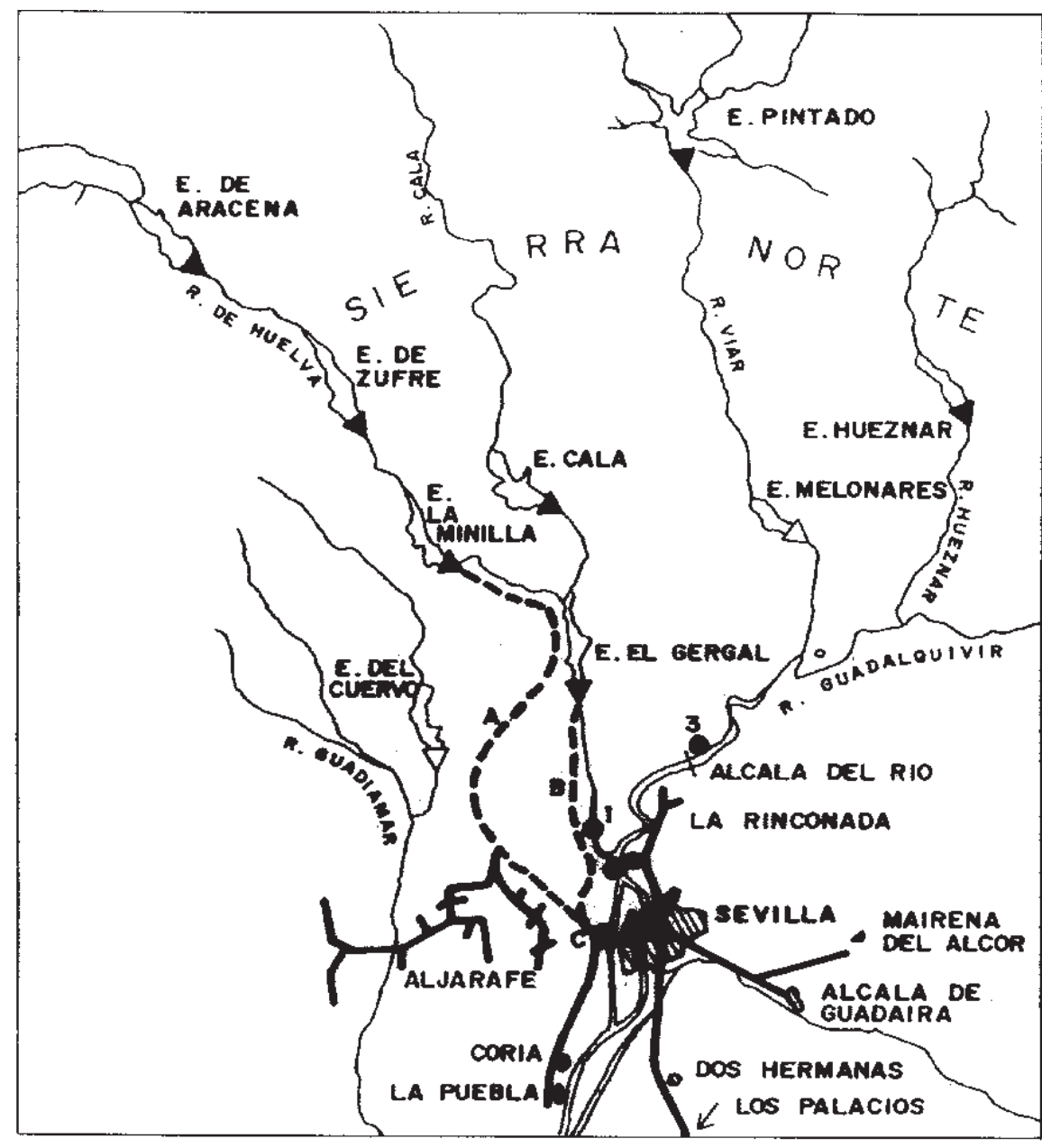

- $1,2.3$, estaciones de EMERgEncia
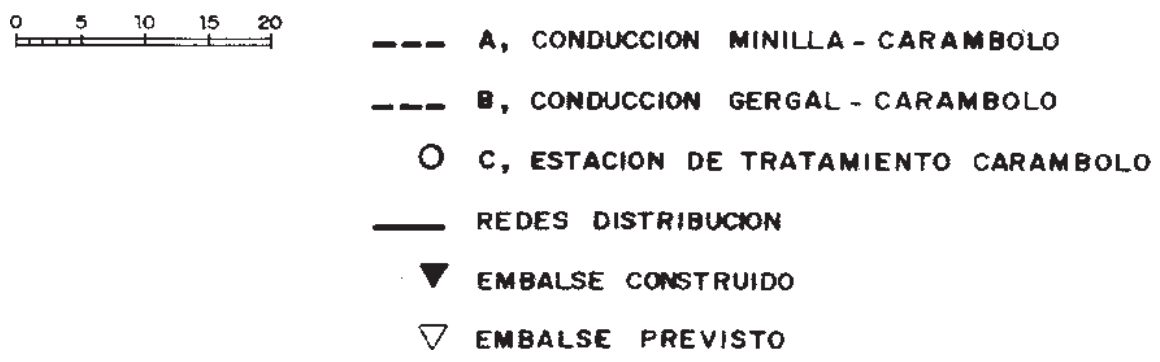

GráfICO 1. Esquema del sistema de abastecimiento a Sevilla y su área de influencia. 


\section{La incertidumbre sobre los recursos disponibles}

Al comenzar la construcción del embalse de Zufre en 1983 — que venía a completar, unido a los ya existentes de La Minilla, Aracena y Gergal, además del hidroeléctrico de Cala, la regulación del río Rivera de Huelva (ver gráfico 1) — la Empresa Municipal de Abastecimiento de Aguas a Sevilla (EMASESA) declaraba que dicho embalse, cuya entrada en servicio estaba prevista para 1987, «permitiría que el fantasma de la sequía sea sólo un recuerdo similar al de las grandes catástrofes de épocas pasadas» (EMASESA, 1983).

Pero no era solamente la Empresa Municipal quien incurría en una apreciaciación que la experiencia posterior ha demostrado equivocada. En fechas más recientes, la Dirección General de Obras Hidráulicas de la Consejería de Obras Públicas de la Junta de Andalucía escribía: «El balance del conjunto, con la reciente puesta en explotación del embalse de Zufre, ofrece en la actualidad una cifra claramente positiva» (JUNTA DE ANDALUCÍA/ CONSEJERÍA DE OBRAS PÚBLICAS Y TRANSPORTES, 1993, p. 84). A continuación se plantea que sólo para situaciones futuras, sin concreción cronológica, habría necesidad de contar con los recursos del embalse de los Melonares y del Huesna (ver gráfico 1).

Por su parte, el tercer organismo implicado, la Confederación Hidrográfica del Guadalquivir (CHG), juzgaba en octubre de 1991 que el sistema de abastecimiento a Sevilla (Sistema de Explotación 13: Sevilla-cuenca del río Rivera de Huelva) mantenía una situación de equilibrio, gracias a la absorción de su ligero déficit con las aguas del mencionado embalse hidroeléctrico de Cala (CONFEDERACIÓN HIDROGRÁFICA DEL GUADALQUIVIR, 1991). En el apartado dedicado a «Problemas de los principales abastecimientos», la CHG mencionaba como problema del abastecimiento de Sevilla y su entorno la necesidad de ampliar la capacidad de conducción. Nada se adivinaba sobre déficit de recursos, por lo que se preveía la asignación del futuro embalse de los Melonares, sobre el río Viar, a la regulación general de la cuenca, no estimándose que dicho embalse fuera necesario para ampliar la garantía del abastecimiento del área de Sevilla.

Sin embargo, la realidad es que a comienzos del año 1993 la situación del abastecimiento del área de Sevilla era extremadamente crítica, tal como se desprende del cuadro siguiente, en el que se compara ese momento con la situación de los dos últimos períodos en los que se adoptaron medidas de emergencia (ver también grafico 2).

\section{Cuadro 1}

RESERVAS DE AGUA A FINALES DEL MES DE FEBRERO EN EL SISTEMA DE ABASTECIMIENTO DE SEVILLA

(en millones de metros cúbicos)

\begin{tabular}{|lccc|}
\hline & $\mathbf{1 9 8 1}$ & $\mathbf{1 9 8 3}$ & $\begin{array}{c}\mathbf{1 9 9 3} \\
\text { (incluyendo Zufre) }\end{array}$ \\
\hline Embalses propios & 38 & 36 & 13 \\
Embalse de Cala & 22 & 34 & 18 \\
\hline TOTAL & 60 & 70 & 31 \\
\hline
\end{tabular}

Fuente: EMASESA. 1985 y 1993. 


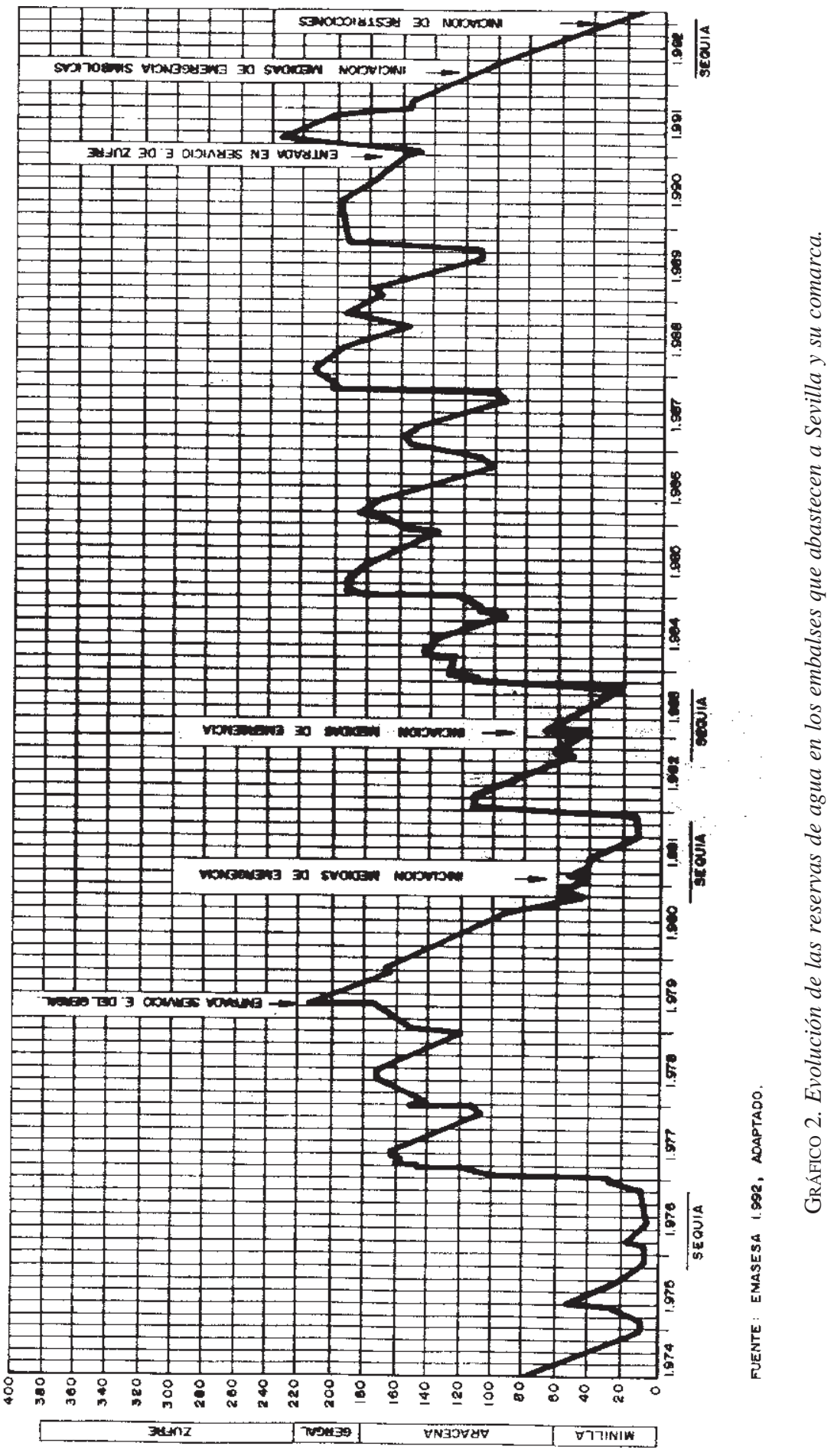


Para entender las razones de esta situación hay que tener en cuenta los siguientes datos:

En primer lugar, el problema del retraso en la entrada en servicio sin limitaciones del embalse de Zufre, a consecuencia de la demora en la construcción de los nuevos accesos a la población del mismo nombre. Este hecho impidió que el embalse, que ya estaba construido, recogiera las escorrentías del invierno 1989-90, extraordinariamente lluvioso (400 litros $/ \mathrm{m}^{2}$ en diciembre del 89). Cuando por fin entró en servicio Zufre, comenzó el actual periodo seco, de manera que las posibilidades del nuevo embalse han quedado inéditas.

Pero junto a esto hay que destacar otro fenómeno que no ha sido afrontado abiertamente por los responsables de la planificación hidrológica: la razón fundamental del contraste entre el diagnóstico de los organismos responsables y la realidad radica en el propio método empleado en el cálculo de la capacidad de regulación de los embalses del sistema; es decir, de la cantidad de agua que dichos embalses garantizan atendiendo a las características del régimen hidrológico de la cuenca que regulan y a las características - tanto en lo que se refiere a la distribución temporal como a los márgenes de déficit admitidos- de la demanda a atender. Este problema, que afecta a la planificación general de las aguas, está siendo debatido en los medios hidrológicos (LÓPEZ ARECHAVALA, 1993). Sin pretender entrar a fondo en este tema, hay que destacar, sin embargo, dos ideas:

a) En el caso de la cuenca del Guadalquivir en su conjunto, el último ciclo seco que se padece desde 1990 ha venido a confirmar la escasa representatividad estadística de las series hidrométricas utilizadas en la elaboración de los estudios de regulación superficial.

b) La aplicación de la nueva normativa dictada en septiembre del 92 por la administración central sobre criterios de garantía para la demanda urbana (MINISTERIO DE OBRAS PÚBLICAS Y TRANSPORTE, 1992) ha servido de ocasión para replantear a la baja la capacidad de regulación del sistema. En esa nueva normativa las déficits admitidos en la atención a la demanda urbana se reducen, por lo que la aplicación de los nuevos criterios al cálculo de la capacidad de regulación redunda en una disminución de la misma. De esta

Cuadro 2

CÁLCULO DE LA CAPACIDAD DE REGULACIÓN DEL SISTEMA DE ABASTECIMIENTO A SEVILLA ANTES Y DESPUÉS DE LA APLICACIÓN DE LOS CRITERIOS DE LA ORDEN MINISTERIAL DE SEPTIEMBRE DE 1992

(en millones de metros cúbicos)

\begin{tabular}{|lccc|}
\hline EMBALSES & $\begin{array}{c}\text { VOLUMEN } \\
\text { DE EMBALSE }\end{array}$ & $\begin{array}{c}\text { CAPACIDAD DE REGULACIÓN BRUTA* } \\
\text { ASIGNADA POR LA C.H.G } \\
(\mathbf{1 9 8 3}, \mathbf{1 9 8 8 , 1 9 8 9 )}\end{array}$ & $\begin{array}{c}\text { ASIGNADA POR EMASESA } \\
\text { (FEBRERO 1993) }\end{array}$ \\
\hline Aracena & 115 & 51 & 35 \\
Minilla & 58 & 40 & 23 \\
Gergal & 35 & 14 & 12 \\
\hline SUBTOTAL & 208 & 105 & 70 \\
\hline Zufre & 168 & 52 & 60 \\
\hline TOTAL con Zufre & 376 & 157 & 130 \\
\hline
\end{tabular}

(*) A la capacidad de regulación bruta hay que restar los volúmenes ecológicos mínimos, con un total de $9 \mathrm{Hm}^{3}$. Fuentes: C.H.G., 1983, 1988 y 1989; EMASESA, 1993. 
manera, se pasa a juzgar insuficiente el sistema de regulación que hasta ese momento, como hemos visto, se consideraba adecuado. En el cuadro 2 se muestra como se ha modificado la evaluación de la capacidad de regulación del sistema antes y después de la apliación de la nueva normativa ministerial.

Es decir, sin contar el embalse de Zufre, el sistema de regulación superficial en el que se basa el abastecimiento a Sevilla, siendo físicamente el mismo — los mismos embalses, con el mismo volumen, sobre el mismo río-, pierde, a efectos de contabilidad de recursos disponibles, 35 millones de metros cúbicos, es decir un 33,5\% de su capacidad de regulación, merced a la aplicación de una nueva fórmula en el cálculo de la misma. A la luz de la situación real del abastecimiento, lo discutible no es, evidentemente, la aplicación de esta nueva fórmula, sino que hasta este momento no se hayan empezado a modificar unas evaluaciones que la realidad demostraba sobrevaloradas.

El hecho de que se hayan repetido situaciones críticas en el abastecimiento de Sevilla en los años 76, 81, 83 y a partir del 92 supone una frecuencia de cuatro en 16 años y, como reconoce EMASESA en 1993, «es síntoma evidente de que en este sistema hidráulico algo está fallando» (EMASESA, 1993).

\section{Una gestión del servicio ajena a planteamientos de conservación del recurso}

Las situaciones críticas de 1981 y 1983, que motivaron severas restricciones (las más estrictas se aplicaron de agosto a noviembre de 1981, con corte del suministro desde las 14 h. hasta las 7 h.) generaron unos hábitos de control del consumo de agua en la población, reforzados por un bajo nivel de recuperación del consumo industrial y comercial debido a los débiles niveles de actividad económica en la ciudad. Pese a la incorporación de nuevas localidades al sistema de abastecimiento metropolitano los volúmenes suministrados se mantienen, sensiblemente estables a lo largo de la primera mitad de la década de los 80 (CRUZ VILLALÓN, 1988).

Cuadro 3

EVOLUCIÓN DEL CONSUMO DE AGUA EN EL ÁREA DE SEVILLA ENTRE 1980 Y 1992 (en millones de metros cúbicos)

\begin{tabular}{|cccc|}
\hline AÑO & $\begin{array}{c}\text { VOLUMEN } \\
\text { SUMINISTRDO }\end{array}$ & $\begin{array}{c}\text { VOLUMEN } \\
\text { FACTURADO }\end{array}$ & $\begin{array}{c}\text { RATIO } \\
\text { FACTURADO/ } \\
\text { SUMINISTRADO }\end{array}$ \\
\hline 1980 & 130,8 & 80,7 & 0,62 \\
1981 & 83 & 59,5 & 0,71 \\
1982 & 99 & 66,6 & 0,67 \\
1983 & 96 & 64,6 & 0,67 \\
1984 & 97 & 68,3 & 0,70 \\
1985 & 96,2 & 75,6 & 0,79 \\
1986 & 113 & & \\
1988 & 142 & & 0,64 \\
1990 & 162 & 103,9 & 0,61 \\
1992 & 175 & 107,2 & 0,63 \\
\hline
\end{tabular}

Fuente: Memorias de EMASESA 1985 y 1992. 
Como puede obervarse en el cuadro 3, los niveles de consumo de 1980 no se recuperaron hasta 1988. Sin embargo, desde entonces lejos de mantenerse ese saludable ambiente de control del gasto, los consumos se disparan creciendo en 63 millones de metros cúbicos, es decir un 55\%, en cinco años, entre 1986 y 1991. En 1992 ya comienzan a dejarse notar los efectos de la nueva situación de sequía.

Por lo que se refiere a la relación entre volúmenes suministrados y volúmenes facturados, hay que destacar esa misma tendencia al relajamiento, volviéndose en 1991 a los grandes niveles de no facturados (39\%), que incluyen consumos municipales y oficiales, pero también un alto nivel de pérdidas en la red de distribución.

No se puede desconocer la labor de EMASESA en el terreno del saneamiento (durante la década de los 80 se construyen las cuatro estaciones que depuran las aguas residuales de Sevilla) y en lo que se refiere a la solución de graves problemas de abastecimiento en poblaciones que se han conectado a la red metropolitana (las últimas Los Palacios y Mairena del Alcor). Sin embargo, a la vista de los datos es indiscutible que los años 80 han sido una década perdida en lo que se refiere a control del despilfarro y modernización de los criterios de relación de la población con un recurso escaso y costoso. Más allá de la retórica, poco se ha avanzado en la línea del desarrollo sostenible de los recursos hidráulicos.

Los motivos hay que buscarlos por una parte en esa percepción sobrevalorada de los recursos disponibles que ya se ha destacado; por otra, en el modelo de crecimiento que domina la segunda mitad de la década de los 80 y principios de los 90.

\section{El enfrentamiento entre los intereses agrarios y urbanos}

Desde la entrada en funcionamiento de la Toma de Emergencia III que bombea 6.000 litros por segundo, Sevilla se abastece de aguas del Guadalquivir, bien de forma exclusiva o bien mezclándola con los recursos que aún conserven los embalses. Mientras tanto, la zona regable del Viar almacena en su embalse del Pintado* una cierta cantidad de agua de excelente calidad $\left(77,4 \mathrm{Hm}^{3}\right.$ el 10 de marzo de 1994).

Ya en la sequía de 1976 se llegó a un acuerdo temporal con los regantes del Viar para usar agua del Pintado en el abastecimiento a la ciudad, cuando EMASESA pagó 21 millones de pesetas a la Comunidad de Regantes del Viar por el trasvase de algo más de 5 $\mathrm{Hm}^{3}$ (CRUZ VILLALÓN, 1988). En la siguiente sequía, 1981, EMASESA solicitó auxilio al MOPU, que acordó la urgente ejecución de una toma en el río Guadalquivir con una capacidad de 1.750 litros por segundo con el fin de suministrar agua del río a los regantes del Viar para compensar los caudales que el Pintado pudiera facilitar a Sevilla. Con ocasión de la crisis actual se han desarrollado negociaciones entre la empresa abastecedora y la comunidad de regantes, que han vuelto a plantear los siguientes temas de interés:

- La institución del dominio público hidráulico excluye la posibilidad de una solución mercantil del problema, a través de una transacción directa entre usuarios.

\footnotetext{
CARACTERÍSTICAS DEL EMBALSE DEL PINTADO

Fecha de entrada en servicio: $\quad 1951$

Volumen:

$202 \mathrm{Hm}^{3}$

Capacidad de regulación:

$100 \mathrm{Hm}^{3}$

Destino:

Potencia instalada:

Producción media anual:

Propietario:

Regadío y energía eléctrica

$18.800 \mathrm{Kw}$

70 mill. Kwh

Administración central
} 
- Sin embargo, la legislación de aguas establece la prioridad de los abastecimientos a poblaciones, incluyendo en su dotación la necesaria para industrias de poco consumo de agua situadas en los núcleos de población y conectadas a la red municipal (art. 58.2 de la Ley de Aguas, 1985).

- Esta misma legislación establece que toda concesión está sujeta a expropiación a favor de otro aprovechamiento que le preceda según el orden de preferencia, con la compensación (justiprecio) que proceda a cargo de beneficiario (arts. 162.2 y 166.2 del Reglamento de Dominio Público Hidráulico), o bien a la sustitución de la totalidad o parte de los caudales concesionales por otros de distinto origen, con el fin de racionalizar el aprovechamiento del recurso (art. 59.3 Ley de Aguas).

- Ya en 1958 se construyó una estación elevadora capaz de suplementar la dotación de la zona regable en $1,5 \mathrm{~m}^{3} / \mathrm{sg}$. con agua tomada en el Guadalquivir. Con ello se posiblita independizar el riego de la parte final, ocupada por la vega de La Algaba, de 1.360 Ha., segregándola del resto de la zona (CONFEDERACIÓN HIDROGRÁFICA DEL GUADALQUIVIR, 1964). Con las instalaciones construidas en 1981 y las tomas de Emergencia III de 1993 es técnicamente posible restituir al sistema de riegos del Viar el caudal que sea preciso detraer para suplir el actual déficit de la demanda urbana (EMASESA, 1993).

Es evidente que nos encontramos ante un caso en el que las medidas de flexibilización del sistema concesional, previstas en el actual marco legal de las aguas, están justificadas. Se trata de buscar fórmulas de desarrollo de un concepto de gran vigencia en el debate hidrológico actual: la reasignación de recursos.

\section{La ausencia de integración de las aguas subterráneas en el abastecimiento urbano}

Cuando en plena crisis del sistema de abastecimiento, el césped de un jardín se conserva verde o una instalación de lavado de automóviles se mantiene en funcionamiento, sus propietarios, para tranquilizar a los ciudadanos, instalan a la vista del público un cártel que dice «Agua de pozo». Quizás sea este fenómeno uno de los más sorprendentes de la trayectoria hidráulica sevillana, que contrasta con la tendencia más acentuada de la planificación hidrológica de los países desarrollados, en los que las aguas subterráneas se protegen como fuente prioritaria de los abastecimientos urbanos de calidad.

La ordenación del territorio de países como Francia, Holanda, Alemania o Italia — por no mencionar a Dinamarca en donde el $98 \%$ de la población se abastece con aguas subterráneas, buena parte de ellas sin ningún proceso de potabilización - tiene en la protección de tales recursos uno de sus objetivos más repetidos.

Es cierto que las condiciones hidrológicas de cada lugar establecen limitaciones específicas. Pero es decididamente poco razonable que el sector noreste del acuífero 27 (a pocos kilómetros del área metropolítana sevillana), de aguas aptas para el consumo de boca sin tratamiento previo, esté satisfaciendo demandas agrarias de cultivos extensivos —en torno a $20 \mathrm{Hm}^{3}$ /año para riegos de arroz, según algunas estimaciones (GIRÁLDEZ CERVERA y otros, 1991)—, con un régimen de explotación que pone en peligro la propia conservación de tales recursos. O que el acuífero de las calcarenitas de Carmona, que surtía tradicionalmente de aguas potables a la ciudad de Sevilla, además de estar sobrexplotado, se haya degradado profundamente (niveles superiores a los $100 \mathrm{mg} / \mathrm{l}$ de $\mathrm{NO}_{3}$ ) impidiendo incluso, en su estado actual, los abastecimientos locales de emergencia (la última población que se ha incorporado al sistema de Sevilla ha sido Mairena del Alcor, que ha abandonado los bombeos del acuífero).

No solamente para hacer frente a estas disfunciones, sino para mejorar las respuestas a los problemas de demanda, el sistema de abastecimiento de Sevilla necesita avanzar en 
la integración de las posibilidades del uso conjunto de recursos superficiales y subterráneos. Hace tiempo están descritas y parcialmente experimentadas las posibilidades de recarga inducida y artificial del acuífero aluvial del Guadalquivir (con una potencialidad estimada en $100 \mathrm{Hm}^{3}$ ) y del ya mencionado de las calcarenitas de Carmona. En relación a este último, el jefe del área de investigación y desarrollo del ITGME afirma que el método de recarga artificial resulta «técnicamente viable y altamente eficaz para mejorar la regulación de los recursos hídricos» (LÓPEZ GETA, J. A. y MURILLO DÍAZ, J. M., 1993).

\section{Balance negativo de la política de información y educación del consumo}

Mención aparte merece el tratamiento del problema ante la opinión pública por parte de las administraciones responsables.

Cuando en febrero de 1992 comenzaron a funcionar las tomas de emergencia del Guadalquivir existentes desde la anterior sequía (tomas de Emergencia I y II), el hecho no trascendió a la opinión pública. Esta recibió una ligera campaña de información cuando el mes siguiente el Ayuntamiento puso en vigor el Plan Especial de Sequía, que no supuso básicamente más que la supresión de agua potable en los servicios municipales de riego y limpieza.

El 13 de septiembre de ese mismo año, el Ayuntamiento puso en vigor nuevas medidas (reducción de la presión durante cuatro horas de la madrugada y llamamiento a la moderación en el consumo). Durante la segunda mitad de septiembre de 1992 se desarrolló una polémica entre la administración central y el Ayuntamiento, con la Expo como telón de fondo. El Secretario de Estado para el Agua, secundado por el Delegado del Gobierno, criticó las medidas mencionadas calificándolas de innecesarias y alarmistas. Sin embargo, a finales del mes de noviembre el Consejero de Obras Públicas y Transportes de la Junta de Andalucía critica al Ayuntamiento de Sevilla por la insuficiencia de las medidas que hasta el momento se habían adoptado para reducir el consumo de agua.

El 1 de diciembre de ese mismo año 1992 se implanta la prohibición del consumo entre las 22 h. y las 6 h., y el 14 de enero de 1993 - tras la reunión de la Mesa de la Sequía que coordina a todas las administraciones implicadas - se amplía la prohibición al período entre las $19 \mathrm{~h}$. y las $7 \mathrm{~h}$. Sin embargo, el 21 de enero se producen unas explosivas declaraciones del Secretario de Estado para el Agua anunciando una próxima normalización del servicio. El 23 del mismo mes, la Comisión Informativa de Medio Ambiente del Ayuntamiento (incluido el partido del Gobierno) rechaza por unanimidad las anteriores declaraciones. Tres días después, EMASESA hace público un comunicado en el que, entre otras cosas, manifiesta que desconoce las nuevas medidas que el Ministerio propone. Ese mismo día, el portavoz del Consejo de Gobierno de la Junta de Andalucía respalda la necesidad de las restricciones, a la vez que confirma las declaraciones del Secretario de Estado en el sentido de que las nuevas obras van a garantizar el «abastecimiento regular de Sevilla». El 29 de ese mismo mes de enero vuelve a reunirse la Mesa de la Sequía presidida por el Secretario de Estado, quien ratifica que las obras anunciadas (toma de emergencia III, en Alcalá del Río) no son una solución provisional, sino que «suministrarán a Sevilla toda el agua que necesite en condiciones normales».

La realidad es que las nuevas tomas de Alcalá del Río, que pueden proporcionar hasta $6 \mathrm{~m}^{3} / \mathrm{sg}$. del Guadalquivir, constituyen una mejora del dispositivo de emergencia, pero su entrada en servicio no se puede plantear como una «regularización», «normalización»o situación de «riesgo cero» del abastecimiento de Sevilla, como las ha presentado la administración central. El agua del río rebasa en algunos parámetros los límites permitidos y ha 
sido precisa la autorización especial de la administración sanitaria para su utilización, atendiendo precisamente a la situación de emergencia (ver cuadro 4).

Cuadro 4

EVOLUCIÓN DE LA CALIDAD DEL AGUA EN SEVILLA

\begin{tabular}{|lccc|}
\hline Parámetros & $\begin{array}{c}\text { Noviembre 91 } \\
\text { (antes de la } \\
\text { sequía) }\end{array}$ & $\begin{array}{c}\text { Noviembre 92 } \\
\text { (sequía } \\
\text { intensa) }\end{array}$ & $\begin{array}{c}\text { Julio 93 } \\
\text { (uso exclusivo } \\
\text { de agua del río) }\end{array}$ \\
\hline $\begin{array}{l}\text { Cond. a } 20{ }^{\circ} \mathrm{C} \\
\text { (us/cm ) }\end{array}$ & 228 & 935 & 1.740 \\
$\begin{array}{l}\text { Cloruros } \\
(\mathrm{mg} / \mathrm{L} \mathrm{Cl})\end{array}$ & 16,9 & 150 & 364,5 \\
$\begin{array}{l}\text { Sodio } \\
(\mathrm{mg} / \mathrm{L} \mathrm{Na)}\end{array}$ & 12 & 92 & 229 \\
\hline
\end{tabular}

Fuente: EMASESA, 1993. Citado por ABC, 14 de noviembre de 1993.

Pasado el verano, el 17 de noviembre de 1993, el Alcalde de Sevilla publica un Bando en el que, considerando que las lluvias de otoño han supuesto un alivio importante, deja «sin efecto todas las medidas restrictivas de consumo de agua» en vigor. A partir de ese momento, se plantea una nueva polémica en la que, en este caso, son los grupos de la oposición municipal y las instituciones provincial y autonómica las que critican el levantamiento de las restricciones y el cese de la utilización de las tomas de emergencia. Ya en la primavera de 1994, arrecia la polémica sobre la mayor o menor urgencia de poner en funcionamiento dichas tomas.

Es de suponer que todo este conflicto, contradicción y descoordinación entre administraciones haya tenido una repercusión negativa sobre el proceso de toma de conciencia de los ciudadanos acerca de la gravedad de la situación y sobre la credibilidad de las instituciones. La tasa de ahorro que a finales de 1992 había alcanzado el 35\% en relación a los consumos anteriores a la crisis, cae al $15 \%$ un año más tarde.

\section{Necesidad de concretar un verdadero programa de gestión de la demanda}

Nadie duda que hay un largo camino que recorrer en el terreno del ahorro y la racionalización del uso del agua:

- Mejora de la eficiencia de las redes de distribución de agua potable, que incluya auditoría, detección y reparación de fugas.

- Autorización del contador individual y tarifas incentivadoras.

- Nuevos sistemas de aplicación del agua doméstica (electrodomésticos de bajo consumo, cisternas de bajo volumen, alcachofas pulverizadoras).

- Adaptación de los modelos de jardinería a las condiciones climatológicas.

- Creación de doble red en relación con las distintas calidades del agua.

Todas ellas son medidas de gestión de la demanda que requieren tiempo e importantes inversiones; pero en esa dirección se mueve, indudablemente, la gestión del agua en los países más avanzados afectados por la escasez del recurso. 
Por poner un ejemplo que sirva de referencia realista: en el Plan de Desarrollo Hidráulico del Estado de Texas actualmente vigente se manejan escenarios de 15, 21 y 30\% de ahorro en el uso doméstico de agua por habitante a largo plazo, basándose en una metodología que incluye el estudio de tendencias históricas, efectividad potencial de las técnicas de conservación, modelos locales de uso del agua, experiencias de medidas de ahorro precedentes, efectos económicos potenciales sobre el sistema (tarifas a usuarios, repercusiones en consumos energéticos y tratamientos de potabilización y depuración) y efectos potenciales sobre necesidades infraestructurales futuras (TEXAS WATER DEVELOPMENT BOARD, 1992). Estas cifras contrastan con el 5\% de reducción de las pérdidas en las redes de conducción que se establece como objetivo en le Anteproyecto de Plan Hidrológico Nacional. Es de destacar, que la Junta de Andalucía considera bajo el objetivo mencionado.

En el Plan de Texas, se generalizan para todo el Estado medidas ya experimentadas que permiten una disminución del agua no facturada desde el actual 15-20\% al 5-10\% En el sistema de Sevilla el porcentaje de agua no facturada ha pasado de un mínimo del $21 \%$ en 1985 a un máximo del 37\% en 1992 (EMASESA, 1985 y 1992).

Dada la inercia de los modelos de gestión hidrológica vigentes, la alternativa al déficit que la administración ofrece actualmente es la de apurar las posibilidades de regulación superficial que aún restan, de crecientes costes económicos. En el caso del abastecimiento a Sevilla, la actual crisis ha relanzado dos proyectos de embalses, ambos con importantes afecciones ambientales. Por una parte, el embalse de Melonares en el río Viar, aguas abajo del ya existente del Pintado; por otra, el embalse del Cuervo, en el río Guadiamar, que completaría la artificialización del régimen del curso que antaño ocasionaba la inundación de la marisma de Doñana.

Todo este debate, incluido el que se refiere a las posibilidades no aprovechadas de los recursos subterráneos y a la reasignación de recursos del regadío al abastecimiento, no tiene un planteamiento racional si no es en el marco de una metodología que permita el análisis coste/beneficio para la sociedad (y complementariamente, para la empresa de abastecimiento y para los usuarios individuales) de las alternativas en presencia.

Más allá de la intuición y percepción subjetiva, los conceptos que, como mínimo, habría que hacer explícitos con la mayor concreción serían los siguientes (GOLVANO SACRISTÁN, 1993):

- Costes evitables con las medidas de gestión de la demanda, incluyendo los costes variables de las instalaciones existentes y los costes totales de las infraestructuras que se puedan evitar o retrasar.

- Externalidades de cada una de las alternativas consideradas. Como es sabido, la evaluación de este factor presenta muchas dificultades, sin embargo de su nivel de precisión depende en buena parte la solvencia del debate.

- Reducción del consumo, que tiene una repercusión contraria sobre empresa («intrínsecamente» interesada en no reducirlo) y usuarios.

- Coste de inversión en tecnológía alternativa, tema sobre las aproximaciones actualmente existentes son muy discrepantes. El escaso desarrollo que de momento el tema está teniendo en España se orienta hacia la modernización de regadíos, sin duda de mucha mayor repercusión cuantitativa y que, a través de la posible reasignación de recursos, incumbe también al problema de los abastecimientos urbanos.

-Variación de costes de operación y mantenimiento (costes energéticos, de potabilización y depuración que, generalmente, disminuirán).

- Costes del programa de gestión, que incluyen los de departamentos de estudios especializados (son necesarios conocimientos profundos de cómo, para qué en qué canti- 
dad usan el agua los diferentes tipos de consumidores) y costes de difusión y promoción.

- Subvenciones, puesto que, teniendo en cuenta que el coste del agua es bajo para los usuarios y que el período de amortización de las inversiones es muy corto, el valor actualizado neto de la reducción del consumo no superará, generalmente, el coste de inversión en tecnologías alternativas.

Todo el análisis debe efectuarse para diferentes escenarios que contemplen modificaciones en las variables más significativas (hidrología, evolución de la demanda, costes de las tecnologías base y alternativa y resultados de los programas de gestión de la demanda).

\section{Bibliografía y documentación citada}

CONFEDERACIÓN HIDROGRÁFICA DEL GUADALQUIVIR (1964): Guadalquivir. Años 19391963, Sevilla.

CONFEDERACIÓN HIDROGRÁFICA DEL GUADALQUIVIR (1991): Proyecto de Directrices del Plan Hidrológico de la cuenca del Guadalquivir. Sevilla.

CRUZ VILLALÓN, J. (1988): «Abastecimiento y consumo de agua en el área de Sevilla» en GIL OLCINA, A. y MORALES GIL, A. (coord.): Demanda y economía del agua en España. Alicante, Caja de Ahorros del Mediterráneo/Diputación Provincial de Alicante.

EMASESA: Memorias Anuales de los años 1983, 1985 y 1992.

EMASESA (1993): Informe al Consejo de Administración. Sevilla, 9 de febrero de 1993, documento mecanografiado.

GIRÁLDEZ CERVERA, J.V. y otros (1991): Informe de las extracciones de agua para uso agrícola del acuífero Almonte-Marismas, Departamento de Agronomía/Universidad de Córdoba.

GOLVANO SACRISTÁN, E. (1993): «Posibilidades de la aplicación de la Planificación al Mínimo Coste en las empresas concesionarias del servicio de suministro de aguas», Revista de Obras Públicas, núm. 3.320, abril 1993, pp. 33-40.

JUNTA DE ANDALUCÍA/CONSEJERÍA DE OBRAS PÚBLICAS Y TRANSPORTES (1993): Bases para la política hidráulica de Andalucía. Sevilla.

LÓPEZ ARECHAVALA, G. (1993): «Datos de base para los estudios hidrológicos» en Las aguas subterráneas en España. Importancia y perspectivas, Instituto Tecnológico Geominero de España (IGTME)/Real Academia de Ciencias Exactas, Físicas y Naturales, pp. 49-67.

LÓPEZ GETA, J.A. y MURILLO DÍAZ, J.M. (1993): «Recarga de acuíferos y reutilización de recursos hídricos» en Las aguas subterráneas. Importancia y perspectivas, I.T.G.M.E/Real Academia de Ciencias Exactas, Físicas y Naturales, pp. 233-254.

MINISTERIO DE OBRAS PÚBLICAS Y TRANSPORTES (1992): Orden de 24 de septiembre de 1992 por la que se aprueban las instrucciones y recomendaciones técnicas complementarias para la elaboración de los Planes Hidrológicos de cuencas intercomunitarias. BOE núm. 249, viernes 16 de octubre.

MORAL ITUARTE, L. del (1991): La obra hidráulica en la cuenca baja del Guadalquivir (siglos XVIII-XX). Gestión del agua y organización del territorio, Sevilla, Consejería de Obras Públicas y Transportes/Universidad de Sevilla/Ministerio de Agricultura, Pesca y Alimentación.

TEXAS WATER DEVELOPMENT BOARD (1992): Water for Texas. Today and tomorrow. Recommendations for the 1992 update of Texas water Plan. Austin (Texas). 